\title{
Time is of essence
}

\author{
Changing the horizon of travel planning
}

\author{
Åsa Nyblom \\ Division of Environmental Strategies Research \\ Centre for Sustainable Communications \\ KTH Royal Institute of Technology \\ Stockholm, Sweden \\ anyblom@kth.se
}

\author{
Elina Eriksson \\ School of Computer Science and Communication \\ Centre for Sustainable Communications \\ KTH Royal Institute of Technology \\ Stockholm, Sweden \\ elina@kth.se
}

\begin{abstract}
Recent years have seen a rapid growth of ICT-services related to the transportation area, and for example Advanced Travel Information Services (ATIS) have been proposed as one means to achieve a decrease of environmental impacts of transportation through behavioural change. However, empirical studies have shown that travel planning in every day life is a fragmented, distributed and fluid process, that is not as enclosed in time and space, or delimited in its content, as today's ICT-services for travel planning imply - in spite of smart and mobile solutions. Hence, in this paper we discuss the role of planning and coordination in the timeconstrained travel-choices of everyday life, together with the question of which time horizons and what decisions that are part of today's travel planning services. Furthermore we will also sketch how travel planners of tomorrow, linked with other ICT-services, could be designed in order to stretch the horizon of assistance so that they are better equipped to support travel planning processes in everyday life. We will also critically discuss the role of travel information services and ICT as being a part of the work for more sustainable mobility.

Index Terms-Everyday practices, ICT, travel information services, travel planning, user-centred perspective
\end{abstract}

\section{Introduction}

There have been a undeniable growth of ICT-services related to the transportation area, and Advanced Travel Information Services (ATIS) [1] have been proposed as a means to change environmentally burdening traffic behaviour. The aim is to make people use public transport to a higher degree and/or to choose active transport alternatives (biking and walking) instead of driving the private car. The ICTservices are proposed to do this mainly by providing people with better, real-time and more accessible travel information [2-4]. However, the expectations of travel information services ${ }^{1}$ (in the following also denoted travel planners) have often been

\footnotetext{
${ }^{1}$ Travel information services or travel planners are ICT based systems giving information on travel choices. Most often this is focused on public

transportation and the information is given through timetables and possibilities to search for a travel route from one place to another.
}

based on models of travel choice and travel behaviour that tend to overemphasise the impact and importance of information and an individualistic perspective $[5,6]$.

Earlier studies [7] have shown that travel planning practice - what planning travel is, and looks like, in people's everyday lives - differs from the simplistic models underpinning most of today's travel planners, that is to, in advance, plan a trip from point $\mathrm{A}$ to point $\mathrm{B}$. Therefore one can argue that the conceptualization of travel planning embedded in the design of travel information services of today, and in parts of the literature, is not reflecting the actual travel planning practice of everyday life. In this paper, we will develop these arguments further, and connect the empirical findings with ICT solutions in general and travel planners in particular. We argue in this paper that there is a need for shifting the horizon of what travel planning is; of when and where it is done, and what factors are included. Thereafter we need to shift the horizon of assistance of tomorrow's travel planners accordingly, if these are to be better equipped to support travel planning processes in everyday life and support environmentally sustainable transport choices.

The article sets out by giving a brief account of the theoretical background and foundation of the article, followed by a description of methodology used for obtaining and analysing the empirical material. Thereafter we move onto the discussion of what separates the common conceptualization of the travel planning process with the planning practice found in empirical studies; briefly discuss the role of planning in timeconstraint realities for mode choices affecting the environmental sustainability of transport; and sketch out how travel planners of tomorrow could expand their horizon of assistance to be better equipped to support travel planning processes in everyday life. Finally, a discussion of the role for travel information systems in the work towards sustainable travel is undertaken.

\section{Background and theoretical framework}

Building on the insights from both practice theory, and STS $^{2}$-studies such as actor network theory, travellers are seen as agents in socio-material networks where the actors (both

\footnotetext{
${ }^{2}$ Science and Technology Studies (STS).
} 
human and non-human) are always interdependent, and behaviour is worked out in practice [8,9]. Consumption of mobility is seen as stemming from shared social norms of what constitutes normal behaviour, normal and attractive ways of life etc., rather than something that is freely chosen by autonomous individuals as a result of their individual attitudes, values and beliefs $[10,11]$. The field study underpinning this article was approached from a practice theory point of view, where the question of what travel planning comprises and how it is done are interdependent - what a practice is depends on how it is done, by whom it is performed, where, when and with what [12]. Analysis of the empirical material from the field study of travel planning practice concluded that the sources of travel information used by people consists of both analogue, ICT-based and informal information sources. These different sources are used in parallel and are often interdependent [7]. Travel information is in the following used to denote all types of information used in travel planning purposes. Formal travel information is denoting information from travel information services, traffic signage, traffic radio etc.

Previous research has also concluded that for most trips, formal travel information is not used [13] and that the application of habits and heuristics limits the need for information [14, 15]. Formal travel information is mostly sought for trips that are long distance [16], unfamiliar, arrival time-sensitive, or unpredictable in some way [2, 6], and when using public transport [17].

\section{Methodology}

The empirical material used in this paper was collected in the context of a research project with the aim of understanding how travel information is utilised in everyday practice and how processes of change in travel habits evolve. Part of the project has been presented in a previous paper [7]. Empirical material was collected first in a small pilot study of 5 interviews, and in a following qualitative field study in a city district of Stockholm. For the field study 19 participants, either working or living in the district, were recruited through personal and professional networks, or through advertisements and on-site recruitment. We sought for people in the process of changing their travel arrangements in some way, e.g. through moving house, starting a new job, having children, getting a smartphone, changing economic status. This was partly done to capture different kinds of changes of travel habit/practice, but it was also a means to overcome the unconscious quality of the frequent routine practices associated with everyday mobility, since everyday practices tend to be more visible in a process of change [18]. Participants of different ages and backgrounds, and with different travel patterns and travel options in their daily life were sought after in order to incorporate as many different narratives as possible. The participants selected ranged from young students working part-time to senior citizens leading active lives.

An explorative method, inspired by the cultural probe methodology [19, 20] and similar to the diary-photo diaryinterview method described by Latham [21], was used. Probe kits containing two assignments - a pre-travel/post-travel diary, and a photo-assignment - were developed to help participants explore and document the planning and choices connected to their everyday mobility. This exploration exercise was designed to make the practice of planning more "visible" to participants. During the interview, the records of the pretravel/post-travel diary and the photographs served as points of departure for the conversation. The in-depth interviews were semi-structured in character. The recorded interviews were transcribed and analysed together with the diaries and photographs. Themes related to the research questions were identified in the material and developed during analysis. All participant names mentioned in this paper are fictitious.

\section{Planning travel in theory and practice}

From where are you going? Where to? When do you want to depart/arrive? These are the typical questions asked by travel information systems of today. Embedded in the system's 'view' of planning, are basically the starting-point, end-point and the timeframes between departure and arrival. The focal point is the single A to B journey, and planning is something you primarily do in order to choose and start your trip. The system's 'view' might also consider price and comfort parameters of different options as important factors in the planning process. This view is prevalent in literature on advanced travel information systems:

\section{Before starting a trip, people determine key parameters such as travel purpose, the time frame, price expectations, or preferred means of transport. People can use web-based travel planners in order to get information and then plan how to travel from point $A$ to point B. [22]}

However, recent studies [7] suggests that travel planning practice in everyday life is not usually structured in this way. Planning is not naturally limited to one occasion of planning, or restricted to the planning of one single trip at the time. Instead travel planning is a process undertaken little by little, squeezed in between doing other things, where final decisions are not always taken until the last minute - or when the deadline for some of the options has already passed. Planning travel is tightly connected to the overall scheduling of activities and thus spanning much larger time frames than the single trip. Planning a business trip may e.g. involve considerations related to the actual workday and to afternoon and evening chores. Moreover, the same planning might also extend further in time and relate to e.g. weekend activities, and to the planning of next week's work. Planning travel also includes considerations of issues such as needed preparations (things you need to do before departure/bring with you on the trip); managing vehicles and equipment in time and space (to get the bike/car home again, or safely parked) the weather (current and coming); social relations (considering different travel options' relation building or 'conflict-triggering' potential); social norms (e.g. of 'good parenting' or 'proper behaviour on public transport'); and health issues (wanting exercise or not managing stairs/luggage). In this view, travel planning is not limited to one journey, but rather the main issue is managing your life, 
and travel planning in some sense becomes a sub-practice to the overarching life scheduling. We argue that this has important implications for design of ICT solutions with the aim of supporting sustainable travel planning. In this paper we will focus the discussion around considerations concerning 1) the blurring of phases, and 2) the change of temporal horizon in and of travel planning.

\section{A. The blurring of phases}

In the literature on Advanced Travel Information Systems (ATIS) the travel planning process is usually divided into two different phases: pre-trip and on-trip, [1, 22]. Pre-trip planning is seen to consist of planning a trip from A to B considering questions as trip purpose, timeframes, mode choice and price; whereas post-trip planning is related to the execution of the trip. Rehrl et al. [22] for example mention information and orientation needs on the selected route, between the different modes of transport, and from the final public transport stop to the actual destination. Taking the behaviour changing potential of travel information services as a point of departure Kramers [23] instead presents a three-phase model of planning, where the different phases pre-trip, on-trip and, added, post-trip, are connected to the different needs in, and functions of planning in these phases. Kramers states that "The needs are primarily for planning before the trip, optimization of route choice during the trip and refinement after the trip" [23 p. 3].

From a technology point of view, distinct phases are of course easier to design for. Empirical evidence suggests however that the travel planning practice of everyday life stubbornly resists conforming to these phases. For example, the very basic form of travel planning, thinking ahead, i.e. the process of laying out options concerning the order of tasks, travel routes, travel mode, trip times, timeframes and needed preparations to scrutiny [7] can be undertaken everywhere and anytime, and is thus not confined to any distinct planning phases. This 'thinking ahead process' is fundamental for identifying uncertainties and gaps of knowledge that might need to be attended to by using external information. The basic travel planning process and the task of filling gaps of knowledge by obtaining more information, could of course take place 'pre-trip', and perhaps sometimes occur in a structured process prior to departure as suggested by [23] and [22]. But the planning of a job-trip might just as well be started the day before on the way home from work, and travel information might be obtained in hurried bits and pieces during the workday. Both in the form of advice by phone from the person one is meeting, and by using travel information services on the Web [7].

From an ICT point of view, a travel information system supportive of this travel planning reality with its blurring of trip-phases would need to support remembering of prior information or knowledge, helping the user keep track of the bits and pieces of the travel planning that has been done so far.

\section{B. Change of Temporal Horizon}

Time is imperative when considering travel and Rehrl, et al. [22] argue that time frames of the (A to B) trip is one of the key parameters in 'pre-trip' travel planning decisions. Although this is accurate, it is only one of several time frames important for travel planning. Time is of essence, and we will here discuss three forms of temporal horizon changes that should be considered when designing new travel information systems: 1) temporal horizon of, and for planning travel 2) inclusion of preparations for the journey in the planning's temporal horizon; and 3) time-slippage in the planning process leading to less sustainable choices. These three are inherently interlinked, both internally and with the blurring of phases, but we are here trying to separate them for clarity of discussion.

\section{a) Temporal horizon - of planning and for planning}

The two perhaps most important shifts of horizons needed in travel information systems have to do with temporal horizons of travel planning. Both of planning itself, seeing travel planning as an over time extended practice; and for planning, acknowledging that the time frames important in planning travel greatly extends the timeframes of a single trip. The travel planning process - undertaken little by little and squeezed in between doing other things - is of course affected by the schedule of the day, in when, and where the planning is made. Furthermore, the planning of a trip is tightly connected to the overall scheduling of activities and involve considerations related to the tasks and timeframes of the entire 'trip'-day, but may also involve considerations related to coming week-end and the to-do list of next week. Planning travel is thus depending on the planning of your day, and of your life, and the planning of travel is often in reality inseparable from the overarching planning of the daily activities and life as a whole.

Seeing travel planning as an over time extended practice, implies that the system must support information retrieval and recalling over time, as well as helping the user when replanning is needed. The user can start planning a particular part of the day in the morning, but then need to re-plan it due to subsequent events. Furthermore, the close connection and interdependence of travel planning and overall scheduling of activities - 'planning of life' suggest that the different ICTbased systems aiming at supporting these practices (travel planners, calendars etc.) need to be connected, which will be discussed further in relation to the following topics of preparations in time and time-slippage.

\section{b) Preparations in time}

Another time horizon important in planning is the time needed for preparations. As found in Nyblom [7], preparations for the journey - i.e. what you need to prepare before you leave, what you need to bring with you, what clothes to wear etc. - are intrinsic parts of the planning process. Timeframes for preparations are not part of the time horizon of travel planners of today, as are usually not timeframes for finding, paying, and walking to and from parking [23]. In the literature there are arguments for the inclusion of the latter into multimodal travel information systems [23], but we would argue that the time- 
frames for needed preparations play an equally important part for people in conducting a journey successfully and on time.

Although some types of preparation needs are highly personal, depending on life situation and trip purpose, others are tied to more general aspects as for example the weather. If travelling by active modes as walking or cycling, a change of the weather often requires changes in clothing and attire: you might not be able to use your ordinary bag due to rainfall and perhaps need to pack a change of clothes. The time for doing such preparations is thus an important factor in planning travel and together with the notion of "time-slippage" discussed in the following section, is also is involved in what mode of travel is utilized in the end, ultimately affecting sustainability of transport.

Weather information is today present in smartphone-apps and Internet websites. But to have a role of assistance when planning travel, weather information needs to be connected to the timeframe of the scheduled journey.

\section{c) Time-slippage}

Linked to the issue of needed preparations for a journey is the notion of time-slippage in the planning process. As noted in [7] the deadline for some options can already have passed when the final decision of if, how and when to make a journey is taken: It is already too late to choose the preferred option of going to a business meeting by metro and collect the car at the garage later, and instead the more complicated and stressful and less sustainable - alternative of collecting the car at the garage first, and drive to the business meeting afterwards is chosen.

In such a situation, ICT could be of assistance. A travel information service that has shifted its horizon of assistance to also include the time-frame of needed preparations and "walking-time" to e.g. a metro station may, if connected to the calendar or schedule of the user be able to send a kind reminder just before the needed 'stand up and prepare to get going'point of the first option arrives. This might serve as the extra push needed for choosing this option, even if it means leaving work a little bit earlier. In the same manner, weather information could be added to the connected ICT systems supporting planning travel and planning life, making it possible to give kind alerts in time when weather changes possibly could give rise to the need for more preparation time than usually needed. This might similarly help avoiding timeslippage and thus perhaps more unsustainable travel choices.

This sketched shift of temporal horizon for travel planners and connected ICT-solutions could be a small way of partly counterbalancing the time-shifting and time-storing capabilities of the car, that compared to public transport and active modes like walking and cycling offer increased flexibility and control of the timing of events; You may departure whenever you want, drive along the route of your choice, transport what you like and wear the clothes of your choice - irrespective of weather $[24,25]$. To go by car is many times the option that demands least preparation time before departure, and is therefore the option left when time has passed all others. Having this kind of 'reminder-system' could never give the same flexibility and control to other modes of transport, but in moving the temporal horizon of assistance they can facilitate and push the use of public transport or active modes that individuals already use, but perhaps not as often as they would like. Health arguments - showing the potential for getting the needed number of steps / physical movement per day with different travel options could be another way of reinforcing the 'counter-balancing' effort of travel planners of tomorrow. The presence of health-arguments in travel planning is supported by empiric evidence from our study, exemplified e.g. by Nina on p. 36 [7].

Counterbalancing efforts of ICT will however not succeed in all situations. An important aspect of time important to travel is that an everyday life situation distinguished by feelings of rush and hurriedness, will seriously affect the planning of life and travel in such a way that the time-shifting capabilities of the private car ultimately win the battle if it is present. Many different narratives in our material support this finding. When on maternity leave, both Nina, 32-year-old journalist, and Sandra, nurse aged 32, used public transport and active transports and found it worked well for their daily life. But reentering into work, with a more time-pressed schedule and many sharp times to meet, the car is winning ground in their everyday travel practice, although the car might not be the preferred choice for other reasons [7].

\section{Discussion and conclusions}

In this paper, we have started from earlier empirical findings showing that travel planning is a fragmented, distributed and fluid process, that is not as enclosed in time and space, or delimited in its content, as today's ICT-services for travel planning imply. This has implications for travel information systems aiming at encouraging sustainable travel choices. We argue in this paper that travel information systems need to widen their temporal horizon of assistance to be in line with existing travel planning practice.

First of all, the fragmented and fluid practice of travel planning extending and blurring the boundaries of pre-, on- and post-trip phases, demands travel planner systems to support remembrance of prior information or knowledge, helping the user keep track of the bits and pieces of the travel planning that has been done so far.

Secondly, seeing that from a practice point of view, the temporal frames important for travel planning is not only the time frames of the journey A to B - but also time frames of activities of the same day, the following week-end, and of the week to come - have implications for the future design of travel information systems. The close connection and interdependence of travel planning and the overall scheduling of activities - 'the planning of life', suggests that the different ICT-based systems aiming at supporting these practices (travel planners, calendars, reminder tools etc.) need to be connected.

Thirdly, the time horizon of successful travel planning practice inevitably includes also the time needed for preparations, i.e. what you need to prepare before you leave, what you need to bring with you, what clothes to wear etc. Weather is a factor that for active modes of transport often 
affects time frames necessary for preparation. This time horizon is neither acknowledged nor included in travel planner systems of today. We argue however, that shifting the temporal horizon of assistance of travel information systems could have an effect for sustainable travel choices. This in the sense that travel planners connected to other ICT-tools could help counterbalancing the time-shifting and time-storing ability inherent in cars. Through including the extended and intertwined temporal horizon of 'planning life' by connecting to calendars and reminder systems; and by including the timeframe of needed preparations and 'walking-time' by e.g. including information about relevant weather changes and walking distances; travel planners of tomorrow may be able to send a kind reminder just before the needed 'stand up and prepare to get going'- point of public transport, walking, or cycling options arrives. This might serve as the extra push needed for choosing these options, and may prevent instances of 'time-slippage' - where final travel plans is not made until after the deadlines of sustainable options already have passed.

Importantly, we need to stress that although this work has been user-centred in the sense that our starting point and perspective have been the existing practice in everyday life, the implications found have not been explored through conceptual designs or evaluation. We here only attempted to sketch out the directions of needed developments travel planners in relation to the empirical evidence of travel planning practice. If we want the future travel planning systems to be relevant and useful in practice, these ideas need to be researched more thoroughly. There are also inevitably important questions related to ethics and the right to privacy arising from the kind of connected systems sketched out above to be addressed.

With the focus of practice, some thoughts and reflections on the 'idea' of ICT solutions become more pronounced. First of all, the focus on practice shows that the planning process is much larger than just the trip from A to B. Indeed, the planning process is intrinsically intertwined with lived life, and in order to support this, there is a risk that any single ICT support becomes too large to be useful. Perhaps the ideas presented in this paper must be implemented in several smaller systems aimed at specific goals in the travel planning and life coordination. Connecting scheduling tools with travel planners, moreover using location data to achieve these changes in horizon of assistance, also raises several important questions related to ethics and the right to privacy to be thoroughly explored and discussed.

Furthermore, the empirical material also show that lived life and practice is contingent on so many things outside the ICT solutions' simplified world, that even though an individual would like to become a more sustainable traveller, stress and other life conditions is pushing in another direction. ICT can perhaps to some degree aid, but probably not be a sole solution. As argued by Baumer and Silberman [26] it is easy to build the solution into the formulation of the problem: by looking upon the problem of non-sustainable travel practices from the viewpoint of technology and ICT, we may hide important nontech solutions and complexities. Looking past the 'ICT as a solution frame' set for the paper, we identify for example social relations, social norms and time as important issues in this respect. In the empirical material we saw a clear connection between time-scarce and busy everyday life situations and an increasing reliance on the time-storing and time-shifting capabilities of the private car. This relates to issues like the culture emphasis of busyness as a moral value, present in many western countries [27] and to wider social norms inscribed in people and societies. Previous studies of the role of ICT in everyday life and social practice give reason for caution, showing that ICT on the one hand is helping people to accommodate the increasing pace and uncertainty of everyday life, but on the other fostering dependence on the same devices and upholding these very social norms and ways of life $[11,28$, 29]. Therefore we end with a suggestion for further research focusing on the systemic role of ICT and advanced travel information systems in forming (sustainable?) societal development, travel practices and social norms.

\section{References}

[1] J. L. Adler and V. J. Blue, "Toward the design of intelligent traveler information systems," Transportation Research Part CEmerging Technologies, vol. 6, pp. 157-172, Jun 1998.

[2] S. Farag and G. Lyons, "What affects use of pretrip public transport information? Empirical results of a Qualitative study," Transportation Research Record: Journal of the transportation Research Board, pp. 85-92, 2008.

[3] S. Farag and G. Lyons, "To use or not to use? An empirical study of pre-trip public transport information for business and leisure trips and comparison with car travel," Transport Policy, 2011.

[4] J. M. Sussman, Perspectives on Intelligent Transportation Systems (ITS), 2005.

[5] G. Lyons, "The role of information in decision-making with regard to travel," IEE Proceedings: Intelligent Transport Systems, vol. 153, 2006.

[6] G. Lyons, E. Avineri, and S. Farag, "Assessing the demand for travel information: Do we really want to know?," presented at the European Transport Conference, Leeuwenhorst Conference Centre, The Netherlands, 2008.

[7] Å. Nyblom, "Making plans or "just thinking about the trip"? Understanding people's travel planning in practice," Journal of Transport Geography, vol. 35, pp. 30-39, 2014.

[8] B. Latour, Reassembling the Social: An Introduction to Actornetwork-theory. Oxford: Oxford University Press, 2005.

[9] T. Schwanen, "Managing uncertain arrival times through sociomaterial associations," Environment and Planning B: Planning and Design vol. 35, pp. 997-1011, 2008.

[10] E. Shove, "Rushing around: coordination, mobility and inequality," 2002.

[11] E. Shove, Comfort, cleanliness and convenience : the social organization of normality. Oxford: Berg, 2003.

[12] E. Shove and M. Pantzar, "Consumers, producers and practices: Understanding the invention and reinvention of Nordic Walking," Journal of Consumer Culture, vol. 5, pp. 43-64, 2005.

[13] G. Lyons, E. Avineri, S. Farag, and R. Harman, "Strategic Review of Travel Information Research," London TDT/149 (R201), 2007.

[14] B. Verplanken, H. Aarts, and A. Van Knippenberg, "Habit, information acquisition, and the process of making travel mode

choices," European Journal of Social Psychology, vol. 27, pp. 539560, 1997. 
[15] T. Gärling, D. Eek, P. Loukopoulos, S. Fujii, O. JohanssonStenman, R. Kitamura, et al., "A conceptual analysis of the impact of travel demand management on private car use," Transport Policy, vol. 9, pp. 59-70, 2002.

[16] S. Peirce and J. Lappin, "Why Don't More People Use Advanced Traveler Information? Evidence from the Seattle Area," in Transportation Research Board 83rd Annual Meeting, Washington DC, 2004.

[17] S. Farag and G. Lyons, "Explaining public transport information use when a car is available: Attitude theory empirically investigated," Transportation, vol. 37, pp. 897-913, 2010.

[18] R. Wilk, "The Edge of Agency: Routines, Habits and Volition," in Time, consumption and everyday life: practice, materiality and culture, E. Shove, F. Trentmann, and R. Wilk, Eds., ed Oxford: Berg Publishers, 2009, pp. 143-156.

[19] B. Gaver, T. Dunne, and E. Pacenti, "Design: Cultural Probes," Interactions, vol. 6, pp. 21-29, 1999.

[20] T. Hemmings, A. Crabtree, T. Rodden, K. Clarke, and K. Rouncefield, "Probing the Probes," in Participatory Design Conference, Malmö, Sweden, 2002, pp. 42-50.

[21] A. Latham, "Researching and writing everyday accounts of the city: an introduction to the diary-photo diary-interview method," in Picturing the Social Landscape: visual Methods and the Sociological Imagination., C. Knowles, Sweetman, P., Ed., ed London: Routledge, 2004, pp. 117-131.

[22] K. Rehrl, S. Bruntsch, and H. J. Mentz, "Assisting multimodal travelers: Design and prototypical implementation of a personal travel companion," Ieee Transactions on Intelligent Transportation Systems, vol. 8, pp. 31-42, Mar 2007.

[23] A. Kramers, "Designing next generation multimodal traveler information systems to support sustainabiliy-oriented decisions," Environmental Modelling \& Software, pp. 1-11, 2014.

[24] E. Shove, "Converging Conventions of Comfort, Cleanliness and Convenience," Journal of Consumer Policy, pp. 395-418, 2003.

[25] E. Shove, T. Frank, and R. R. Wilk, Eds., Time, consumption and everyday life: practice, materiality and culture. Oxford: Berg, 2009, p.^pp. Pages.

[26] E. P. S. Baumer and M. S. Silberman, "When the implication is not to design (technology)," presented at the CHI, Vancouver, BC, Canada, 2011.

[27] G. Leshed and P. Sengers, "'I lie to myself that I have freedom in my own schedule': productivity tools and experiences of busyness," in Proceedings of the SIGCHI Conference on Human Factors in Computing Systems, Vancouver, BC, Canada, 2011.

[28] J. Jain, T. Line, and G. Lyons, "A troublesome transport challenge? Working round the school run," Journal of Transport Geography, vol. 19, pp. 1608-1615, 2011.

[29] T. K. Christensen and I. Røpke, "Can Practice Theory Inspire Studies of ICTs in Everyday Life?," in Theorising media and practice, B. Bräuchler and J. Posthill, Eds., ed Oxford and New York: Berghahn Books, 2011. 